This item was submitted to Loughborough's Research Repository by the author.

Items in Figshare are protected by copyright, with all rights reserved, unless otherwise indicated.

\title{
Universal upper critical field of unconventional superconductors
}

PLEASE CITE THE PUBLISHED VERSION

LICENCE

CC BY-NC-ND 4.0

\section{REPOSITORY RECORD}

Zavaritsky, V.N., V.V. Kabanov, and A.S. Alexandrov. 2019. "Universal Upper Critical Field of Unconventional Superconductors". figshare. https://hdl.handle.net/2134/1136. 


\title{
Universal upper critical field of unconventional superconductors
}

\author{
V.N. Zavaritsky ${ }^{1,2}$, V.V. Kabanov ${ }^{3}$, and A.S. Alexandrov ${ }^{1}$ \\ ${ }^{1}$ Department of Physics, Loughborough University, Loughborough LE11 3TU, United Kingdom; \\ ${ }^{2}$ Kapitza Institute for Physical Problems, 2 Kosygina Str., 117334 Moscow, Russia; \\ ${ }^{3}$ Josef Stefan Institute 1001, Ljubljana, Slovenia.
}

(February 20, 2006)

\begin{abstract}
The resistive upper critical field, $H_{c 2}(T)$ of cuprates, superconducting spin-ladders, and organic (TMTSF $)_{2} \mathrm{X}$ systems is shown to follow a universal nonlinear dependence $H_{c 2} \propto\left(T_{c}-T\right)^{3 / 2}$ in a wide range near $T_{c}$, while its low-temperature behaviour depends on the chemical formula and sample quality. $H_{c 2}(T)$ is ascribed to the Bose-Einstein condensation field of preformed pairs. The universality originates from the scaling arguments. Exceeding the Pauli paramagnetic limit is explained. Controversy in the determination of $H_{c 2}(T)$ from the kinetic and thermodynamic measurements is resolved in the framework of the charged Bose-gas model with impurity scattering.
\end{abstract}

74.20.-z,74.72.-h , 74.70.Kn

The upper critical field is one of the fundamental characteristics of type II superconductors. For sufficiently high field, superconductivity is destroyed and the field is uniform in a bulk sample. Continuously decreasing the field superconducting regions begin to nucleate spontaneously at a certain filed $B=H_{c 2}(T)$. In the regions where the nucleation occurs, superconductivity is just beginning to appear, so that the density of supercarriers, $n_{s}=|\psi(\mathbf{r})|^{2}$ is small. Hence, the phenomenological Landau-Ginsburg (LG) (or the microscopic Gor'kov) equation for the order parameter $\psi(\mathbf{r})$ can be linearized to give

$$
\frac{1}{2 m}(\nabla-2 i e \mathbf{A}(\mathbf{r}))^{2} \psi(\mathbf{r})=\alpha \psi(\mathbf{r})
$$

where $\hbar=c=k_{B}=1 . \quad H_{c 2}(T)$ allows for a direct measurement of the most fundamental parameter, the superconducting coherence length, $\xi(T)$, because $H_{c 2}=$ $\phi_{0} / 2 \pi \xi(T)^{2}$ ( $\phi_{0}$ is the flux quantum) [1]. Solving Eq.(1), one obtains the linear $H_{c 2}(T)=-m \alpha / e$ near $T_{c}$ [2] with $\alpha \propto T-T_{c}$ in the Landau theory of the second-order phase transitions. At zero temperature $H_{c 2}(0)$ is normally below the Clogston-Chandrasekhar [3] or the Pauli pair-breaking limit given by $H_{p} \simeq 1.84 T_{c}$ (in Tesla) for the singlet pairing. The limit can be exceeded due to the spin-orbit coupling 团, or triplet pairing, but in any case $H_{c 2}(0)$ remains finite in the framework of the BCS theory. The mean-field BCS approach, Eq.(1), is applied if $\xi(0)>>(6 / n \pi)^{1 / 3}$, where $n$ is the carrier density. Hence, irrespective to the Pauli pair-breaking limit, the zero temperature value of the (BCS) upper critical field should be much less than $\phi_{0}(\pi n / 6)^{2 / 3} /(2 \pi)$, which is about 200 Tesla for a typical carrier density in novel superconductors $\left(n \simeq 10^{21} \mathrm{~cm}^{-3}\right)$.

In cuprates [5 16], spin-ladders [17] and organic superconductors [18] high magnetic field studies revealed a non-BCS upward curvature of resistive $H_{c 2}(T)$. When measurements were performed on low- $\mathrm{T}_{c}$ unconventional superconductors 66, 7, 11, 17, 18, the Pauli limit was exceeded by several times. A non-linear temperature dependence in the vicinity of $\mathrm{T}_{c}$ was unambiguously observed in a few samples [8,11,14 16]. This strong departure from the canonical BCS behaviour led some authors [10, 19, 21] to conclude, that the abrupt resistive transition in applied fields is not a normal-superconductor transition at $H_{c 2}$. Indeed, the thermodynamic determination of $H_{c 2}$ [10,21,23, and anomalous diamagnetism above the resistive transition [24,19] seem to justify such a conclusion. Thermodynamically determined $H_{c 2}$ appears to be linear in the vicinity of $T_{c}$, and much higher than the resistive $H_{c 2}$, in some cases 19,21 exceeding well not only the Pauli limit, but even the ultimate 'BCS' limit mentioned above.

The apparent controversy in different determinations of $H_{c 2}$ needs to be addressed beyond the mean-field approach, Eq.(1). Unconventional superconductors could be in the 'bosonic' limit of preformed real-space pairs, so their resistive $H_{c 2}$ is actually a critical field of the Bose-Einstein condensation of charged bosons, as proposed by one of us 25]. The calculations [26] carried out for the heat capacity of an ideal charged Bose-gas in a magnetic field revealed a remarkable difference between the resistive $H_{c 2}$ and the thermodynamically determined one. While any magnetic field destroys the condensate of ideal bosons, it hardly shifts the specific heat anomaly.

In this Letter, we present a comprehensive scaling of resistive $H_{c 2}$ measurements in a great variety of unconventional superconductors. A universal non-BCS temperature dependence is found in the vicinity of $T_{c}$ while deviations from the universality are observed at low temperatures. We describe these results in the framework of a microscopic model of charged bosons scattered off impurities. Different from the ideal Bose-gas this model predicts two anomalies in the specific heat. The lower temperature anomaly traces the resistive transition in a 
magnetic filed, but the higher one is hardly shifted even by a high magnetic field, as observed [22,23. Based on the microscopic model we argue that the state above the resistive $H_{c 2}(T)$ of unconventional superconductors is the normal state of preformed pairs.

In the bosonic superconductor the mean-field LG equation, Eq.(1) is replaced by the microscopic Schrödinger equation for the condensate wave function 25],

$$
\left[\hat{U}_{s c}-(\nabla-2 i e \mathbf{A}(\mathbf{r}))^{2} /(2 m)\right] \psi(\mathbf{r})=\mu \psi(\mathbf{r}),
$$

where $\hat{U}_{s c}$ is the scattering potential due to impurities and phonons, or the self-energy operator due to interparticle hard-core and long-range correlations [28], and $\mu$ is the chemical potential. Different from the mean-field Eq.(1), it takes fully into account both thermal and quantum fluctuations, but does not allow for a direct determination of $H_{c 2}$. When $H_{c 2}$ is defined as the field where the first non-zero extended solution of Eq.(2) appears, the equation yields a position of the chemical potential at the mobility edge $\mu=E_{c}$, rather than $H_{c 2}$ itself. Then the upper critical field is found using the total number of extended bosons $n_{b}$ above the mobility edge,

$$
\int_{E_{c}}^{\infty} f(\varepsilon) N\left(\varepsilon, H_{c 2}\right) d \varepsilon=n_{b}(T),
$$

where $N(\varepsilon, B)$ is the density of states (DOS) of the Hamiltonian, Eq. $(2)$, and $f(\varepsilon)=1 /[\exp ((\varepsilon-\mu) / T-1]$ is the Bose-Eistein distribution. In the general case $n_{b}(T)$ depends on temperature due to a partial localization of bosons in the random potential.

Applying simple scaling arguments 25] the positive curvature of $H_{c 2}(T)$ near $T_{c}$ and its divergent behaviour at low temperatures follow from Eq.(3). The number of bosons at the lowest Landau level $(n=0)$ is proportional to the temperature and DOS near the mobility edge, $N_{0} \propto B / \sqrt{\Gamma_{0}(B)}$. The collision broadening of the Landau level is also proportional to the same DOS $\Gamma_{0}(B) \propto B / \sqrt{\Gamma_{0}(B)}$. Hence, $\Gamma_{0}(B) \sim B^{2 / 3}$ and therefore the number of bosons at the lowest level is proportional to $T B^{2 / 3}$. The singularity of all upper levels' DOS is integrated out in Eq.(3) so that one can neglect their quantization using the zero field density of states for the levels with $n \geq 1$. Equating the number of bosons with $n=0$ and $n_{b}(T)-n_{b}\left(T_{c}\right)\left(T / T_{c}\right)^{3 / 2}$ (the total number minus the number of thermally excited bosons with $n \geq 1$ ) yields

$$
H_{c 2}(T)=H_{0}\left[n_{b}(T) /\left(\operatorname{tn}_{b}\left(T_{c}\right)\right)-t^{1 / 2}\right]^{3 / 2},
$$

where $t=T / T_{c}$. The scaling constant $H_{0}$ depends on the scattering mechanism, $H_{0}=\phi_{0} / 2 \pi \xi_{0}^{2}$, with the characteristic (coherence) length $\xi_{0} \simeq\left(l / n_{b}\left(T_{c}\right)\right)^{1 / 4}$. Here $l$ is the zero-field mean-free path of the low energy bosons. One obtains the parameter-free $H_{c 2}(T) \propto(1-t)^{3 / 2}$ using Eq.(4) in the vicinity of $T_{c}$, but the low-temperature behaviour depends on the particular scattering mechanism, and the detailed structure of the density of localized states. As suggested by the normal state Hall measurements in cuprates 27] $n_{b}(T)$ can be parameterized as $n_{b}(T)=n_{b}(0)+$ constant $\times T$, so that $H_{c 2}(T)$ is described by a single-parameter expression as

$$
H_{c 2}(T)=H_{0}\left[b(1-t) / t+1-t^{1 / 2}\right]^{3 / 2} .
$$

Parameter $b$ is proportional to the number of delocalised bosons at zero temperature. We expect that this expression applies to the whole temperature range except ultra-low temperatures, where the Fermi Golden-rule in the scaling fails [28. Exceeding the Pauli pair-breaking limit readily follows from the fact, that the singlet-pair binding energy is related to the normal-state pseudogap temperature $T^{*}$, rather than to $T_{c}$ [29]. $T^{*}$ is higher than $T_{c}$ in bosonic superconductors, and cuprates.

The universal scaling of $H_{c 2}$ near $T_{c}$ is confirmed by the resistive measurements of the upper critical field of many cuprates, spin-ladders, and organic superconductors, as shown in Fig.1A. All data reveal the universal $(1-t)^{3 / 2}$ behaviour in a wide temperature region as can be seen in the inset to Fig.1A. Deviations from this law, observed in a few cuprates in a close vicinity of $T_{c}$ were explained in Ref. [8]. The low-temperature behaviour of $H_{c 2}(T) / H_{0}$ is not universal, but well described using Eq. (5) with a single fitting parameter, $b$. This is close to 1 in high quality cuprates with a very narrow resistive transition [8, 14, 16]. It naturally becomes rather small in overdoped cuprates where the randomness is more essential, so almost all bosons are localized (at least in one dimension) at zero temperature. It becomes even smaller in organic superconductors, which might be related to the magnetic field induced dimensional crossover [30] at low temperatures. The scaling parameter $H_{0}$ increases with increasing $T_{c}$, Fig.1B. This is because mean-free path $l$ decreases with doping, while the density of carriers increases, so that the coherence length $\xi_{0}$ becomes smaller in the cuprates with a higher $T_{c}$.

Calculations of the specific heat require the analytical DOS, $N(\varepsilon, B)$ of a particle in the random potential and in the magnetic field. The above scaling suggests that $H_{c 2}(T)$ is not sensitive to a particular choice of the scattering mechanism and approximation, at least in a wide vicinity of $T_{c}$. Hence, one can use the canonical noncrossing approximation for the single-particle self-energy,

$$
\Sigma_{\nu}(\varepsilon)=\sum_{\nu^{\prime}} \frac{\Gamma_{\nu, \nu^{\prime}}}{\varepsilon-\varepsilon_{\nu^{\prime}}-\Sigma_{\nu^{\prime}}(\varepsilon)}
$$

with a particular scattering matrix element squared $\Gamma_{\nu, \nu^{\prime}}=\Gamma \delta_{n, n^{\prime}}, \nu \equiv\left(n, p_{x}, p_{z}\right)$ are the quantum numbers of the Landau problem. This allows us to obtain an analytical result for the DOS, $N(\varepsilon, B)=\Gamma^{-1} \sum_{n} \Im \Sigma_{n}(\varepsilon)$ as 


$$
\begin{aligned}
N(\varepsilon, B) & =\frac{e B}{4 \pi^{2}} \sqrt{\frac{6 m}{\Gamma_{0}}} \sum_{n}\left[\left(\tilde{\varepsilon}^{3}+\frac{1}{2}+\sqrt{{\tilde{\varepsilon_{n}}}^{3}+\frac{1}{4}}\right)^{1 / 3}\right. \\
& \left.-\left(\tilde{\varepsilon}^{3}+\frac{1}{2}-\sqrt{{\tilde{\varepsilon_{n}}}^{3}+\frac{1}{4}}\right)^{1 / 3}\right]
\end{aligned}
$$

with the mobility edge at $E_{c}=e B / m-3 \Gamma_{0} / 2^{2 / 3}$. Here $\Gamma_{0}=0.5(2 \Gamma e B \sqrt{m} / \pi)^{2 / 3}$ is the collision broadening of the lowest Landau level, $\tilde{\varepsilon}_{n}=[\varepsilon-2 e B(n+1 / 2) / m] / 3 \Gamma_{0}$.

$H_{c 2}(T)$ calculated with the analytical DOS, Eq.(7) is almost the same as $H_{c 2}$ in Eq.(5). The specific heat coefficient $C(T, B) / T=d\left[\int d \varepsilon N(\varepsilon, B) \varepsilon f(\varepsilon)\right] / T d T$ calculated with the same DOS and with $\mu$ determined from $n_{b}=\int d \varepsilon N(\varepsilon, B) f(\varepsilon)$ is shown in Fig.2a. The broad maximum at $T \simeq T_{c}$ is practically the same as in the ideal Bose gas without scattering [28]. It barely shifts in the magnetic field. However, there is the other anomaly at lower temperatures, which is absent in the ideal gas. It shifts with the magnetic field, tracing the resistive transition, as clearly seen from the difference between the specific heat in a field and the zero-field curve, Fig. 2b. The specific heat, Fig. 2, is in striking resemblance to the Geneva group's experiments on $\mathrm{DyBa}_{2} \mathrm{Cu}_{3} \mathrm{O}_{7}$ (Fig. 4 and 6 in Ref. 23) and on $\mathrm{YBa}_{2} \mathrm{Cu}_{3} \mathrm{O}_{7}$ (Fig. 1 and 2 in Ref. [22]), where both anomalies were observed.

Within our model, when the magnetic field is applied, it hardly changes the temperature dependence of the chemical potential near $T_{c}$ since the energy spectrum of thermally excited bosons remains practically unchanged. That is because their characteristic energy (of the order of $T_{c}$ ) remains enormous compared with the magnetic energy of the order of $2 e \mathrm{~B} / \mathrm{m}$. In contrast, the energy spectrum of the low energy bosons is strongly perturbed even by a weak magnetic field. As a result the chemical potential 'touches' the band edge at lower temperatures, while having almost the same 'kink'-like temperature dependence around $T_{c}$ as in zero field. While the lower anomaly corresponds to the true long-range order, the higher one is just a trace-'memory' of the zero-field transition. Hence, our microscopic consideration shows that the genuine phase transition into the superconducting state is related to the resistive transition and to the lower specific heat anomaly. The broad higher anomaly is the normal state feature of the bosonic system in the external magnetic field. Different from the BCS superconductor these two anomalies are well separated in the bosonic superconductor at any field except zero one. Hence, the resistive $H_{c 2}$ is the genuine upper critical field, while the field $H^{*}$ determined thermodynamically from the higher anomaly of the specific heat, Fig. 2b, is a pseudo-critical field, unrelated directly to the long-range off-diagonal superconducting order. The absence of significant superconducting fluctuations in the resistivity of the highest quality samples [14, 6, 7, 11, 13] in a wide field interval between the resistive $H_{c 2}(T)$ and $H^{*}$ further justifies the conclusion. A weak diamagnetism observed in a few cuprates above the resistive $H_{c 2}(T)$ curve [24, 19], was explained as the normal state Landau diamagnetism of preformed pairs in the framework of the same microscopic model of charged bosons 31].

Our conclusions are at variance with some others [20], which claim that strongly anisotropic Bi-cuprates remain in the superconducting state well above the resistive $H_{c 2}(T)$. However, thorough analysis [33] of the data used by 20 to support that claim reveals significant contribution from extrinsic effects. These are responsible for the apparent contradiction between the results of 20] and those of the predecessors [8,13,16]. In particular, as shown in [33], the unusual shape of $\rho_{a b}(H)$ [20] could result from the current redistribution in a defective crystal while the Joule heating is likely to be responsible for the non-Ohmic resistance observed in [20]. Moreover, as shown in Fig. 3, when the routine procedure for the resistive $H_{c 2}$ evaluation [8] is applied to reliable in-plane and out-of-plane data obtained on the same samples [9, 32], very similar values of $H_{c 2}(T)$ are obtained from $\rho_{c}$ and $\rho_{a b}$ [33]. This puts into question the last argument of the authors of Ref. [20] who claim that while $\rho_{c}$ is a measure of the interplane tunneling, only the in-plane resistivity represents a true normal state and should be used in the determination of $H_{c 2}$. It is appropriate to mention here that according to the analysis in Fig.1, significant features of the resistive $H_{c 2}$ appear to be robust with respect to the particular component of the resistivity used for its evaluation. Finally, the observation 13. of the negative c-axis magnetoresistance above $T_{c}$ invalidates the main claim of Ref. [20] that it is a signature of the superconducting state.

In conclusion, we have scaled the magnetotransport measurements in many novel superconductors. The unusual upper critical field $H_{c 2}(T)$ has been ascribed to the Bose-Einstein condensation field of preformed pairs. We have introduced a charge Bose-gas model with a particular choice of the scattering potential allowing for the analytical DOS in the magnetic field. In contrast to an ideal Bose-gas model and the BCS theory, this model describes well resistive $H_{c 2}(T)$ and predicts two anomalies in the specific heat. We have shown that the genuine phase transition into the superconducting state is related to the resistive transition and to the lower specific heat anomaly, while the higher one is the normal state feature of the bosonic system in the external magnetic field. Our approach is compatible with a wealth of various experimental observations, the normal pseudogap and the absence of the Hebel-Slichter peak being only a few of them [29].

The authors acknowledge valuable discussions with A. F. Andreev, V. F. Gantmakher, L. P. Gor'kov, A. Junod, W. Y. Liang, J. W. Loram, V. V. Moshchalkov, M. Springford, and G. M. Zhao. The financial support of the EPSRC (Ref.: R/46977) and the Leverhulme Trust (Ref.: F/00261/H) is gratefully acknowledged. 
[1] P. G. de Gennes, Superconductivity of Metals and Alloys, Addison-Wesley Publishing (New York, 1989), p. 195.

[2] Hereafter $T_{c}$ denotes the zero field critical temperature.

[3] A. M. Clogston, Phys. Rev. Lett. 9, 266 (1962);

B. S. Chandrasekhar, Appl. Phys. Lett. 1, 7 (1962).

[4] N. R. Werthamer, E. Helfand, and P. C. Hohenberg, Phys. Rev. 147, 295 (1966).

[5] B. Bucher et al, Physica C 167, 324 (1990).

[6] A.P. Mackenzie et al, Phys. Rev. Lett. 71, 1238 (1993). A.Carrington et al, Phys. Rev. B 49, 13243 (1994).

[7] M.S. Osofsky et al, Phys. Rev. Lett. 71, 2315 (1993).

[8] A.S. Alexandrov et al, Phys. Rev. Lett. 76, 983 (1996).

[9] Y. Ando et al, Phys. Rev. Lett. 79, 2595 (1997).

[10] A. Carrington et al, Phys. Rev. B 54, R3788 (1996).

[11] D.J.C.Walker et al, Phys. Rev. B 51, 9375 (1995). D.D. Lawrie et al, J. Low Temp. Phys. 107, 491 (1997).

[12] Y. Ando et al, Phys. Rev. Lett. 75, 4662 (1995). G.S. Boebinger et al, Phys. Rev. B 60, 12475 (1999).

[13] V.N.Zavaritsky, M.Springford, JETP Lett. 5, 448 (1998).

[14] V.F. Gantmakher et al, JETP 88, 148 (1999).

[15] Y.Dalichaouch et al, Phys. Rev. Lett. 64, 599 (1990). S.H. Han et al, Phys. Rev. B 46, 14290 (1992). M. Suzuki, M. Hikita, Phys. Rev. B41, 9566 (1990). V. F. Gantmakher et al, JETP Lett. 72, 21 (2000).

[16] V.N. Zavaritsky, M. Springford, A.S. Alexandrov, Europhys. Lett. 51, 334 (2000).

[17] T. Nakanishi et al, Int. J. Mod. Phys. 14, 3617 (2000).

[18] I. J. Lee, P. M. Chaikin, and M. J. Naughton, Phys. Rev. B 62, R14 669 (2000).

[19]

H.H. Wen, S.L. Li, Z.X. Zhao, Phys. Rev. B62, 716 (2000).

[20] N. Morozov et al, Phys. Rev. Lett. 84, 1784 (2000).

[21] J. L. Luo et al, cond-mat/0112065.

[22] M. Roulin et al, J. Low Temp. Phys. 105, 1099 (1996).

[23] B. vaz, A. Junod, A. Erb, Phys. Rev. B58, 11153 (1998).

[24] A. Junod et al, Physica C 294, 115 (1998).

[25] A.S. Alexandrov, Phys. Rev. B48, 10571 (1993).

[26] A. S. Alexandrov et al, Phys. Rev. Lett. 79, 1551 (1997).

[27] A. S. Alexandrov, A. M. Bratkovsky, and N. F. Mott, Phys. Rev. Lett. 72, 1734 (1994).

[28] A. S. Alexandrov, W. H. Beere, and V. V. Kabanov, Phys. Rev. B54, 15363 (1996).

[29] A.S. Alexandrov and N.F. Mott, Rep. Prog. Phys. 57, 1197 (1994)

[30] A. G. Lebed, JETP Lett. 44, 114 (1986).

[31] C.J. Dent, A.S. Alexandrov, and V.V. Kabanov, Physica C 341-348, 153 (2000).

[32] Y. Z. Zhang et al, Phys. Rev. B 61, 8675 (2000).

[33] V. N. Zavaritsky, M. Springford, and A. S. Alexandrov, cond-mat/0011192; Physica B 294-295, 363 (2001).

\section{Figure Captures}

Fig. 1. A: Resistive upper critical field (evaluated at $50 \%$ of the transition) of electron/hole doped cuprates, spin-ladders and organic superconductors scaled accord- ing to Eq.(5). Parameter b is 1 (solid line), 0.02 (dasheddotted line), 0.0012 (dotted line), and 0 (dashed line). Inset shows the universal scaling of the same data near $T_{c}$. B: $H_{0}$ versus $T_{c} / T_{c}^{o p t}$, where $T_{c}^{o p t}$ is the critical temperature of the optimally doped material (shown in brackets). Lines are the guide for eyes. The right-hand part of $\mathbf{B}$ shows $H_{0}$ for the compounds where $T_{c}^{o p t}$ is unknown.

Fig.2. Temperature dependence of the specific heat $C(H, T) / T$ (in units of $2 n k_{B} /\left[\pi^{1 / 2} \zeta(3 / 2) T_{c}\right]$ ) of charged Bose-gas scattered off impurities for several fields indicated in the figure $\left(\omega_{H}=2 e B / m\right)$. Fig. 2b: Likewise 2224 shows $C(H, T)-C(0, T)$ and reveals two anomalies: the lowest traces the resistive transition while the highest, $H^{*}$, is the normal state feature.

Fig.3. $H_{c 2}(T)$ obtained from $\rho_{a b}$ (solid lines) and $\rho_{c}$ (dashed lines) of the same sample [9, 32. 


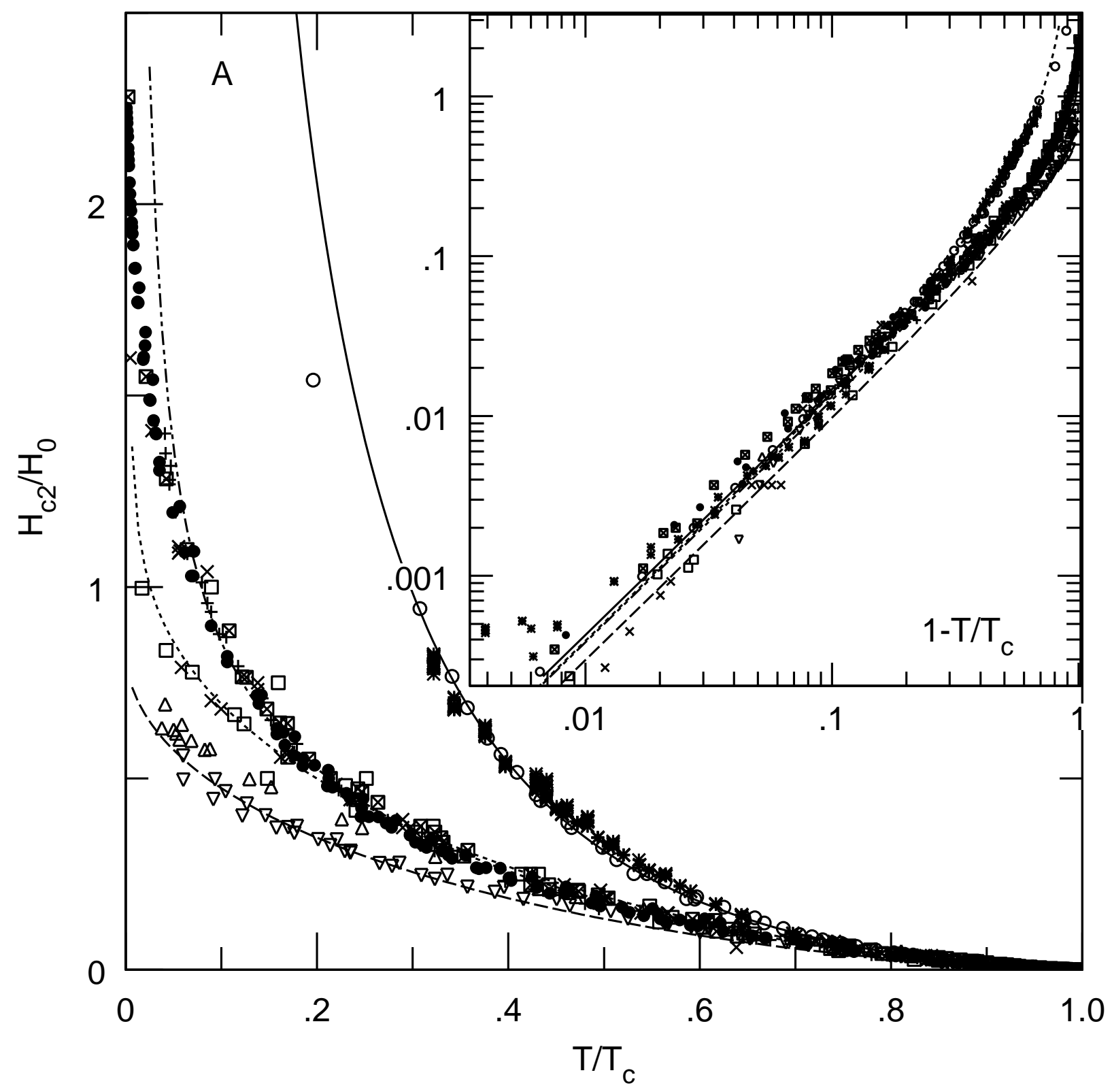




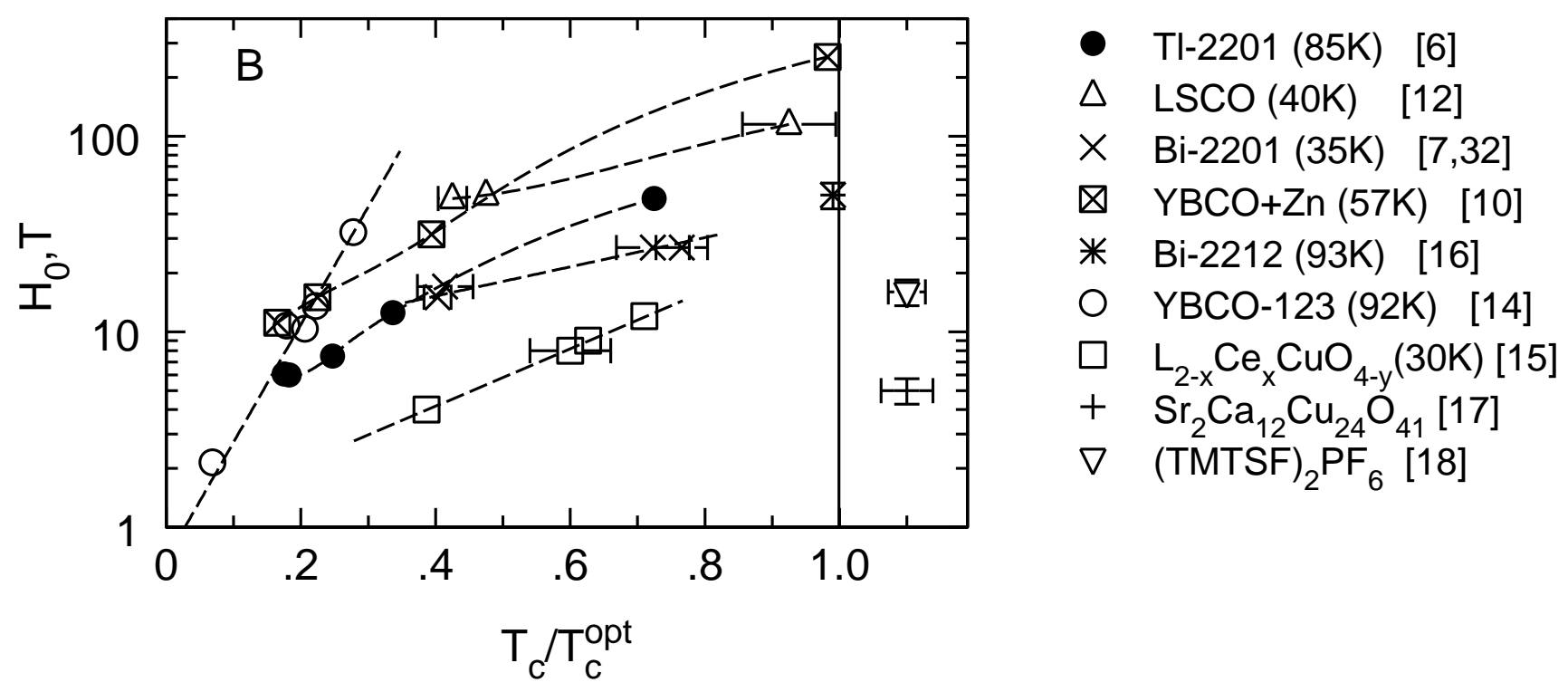




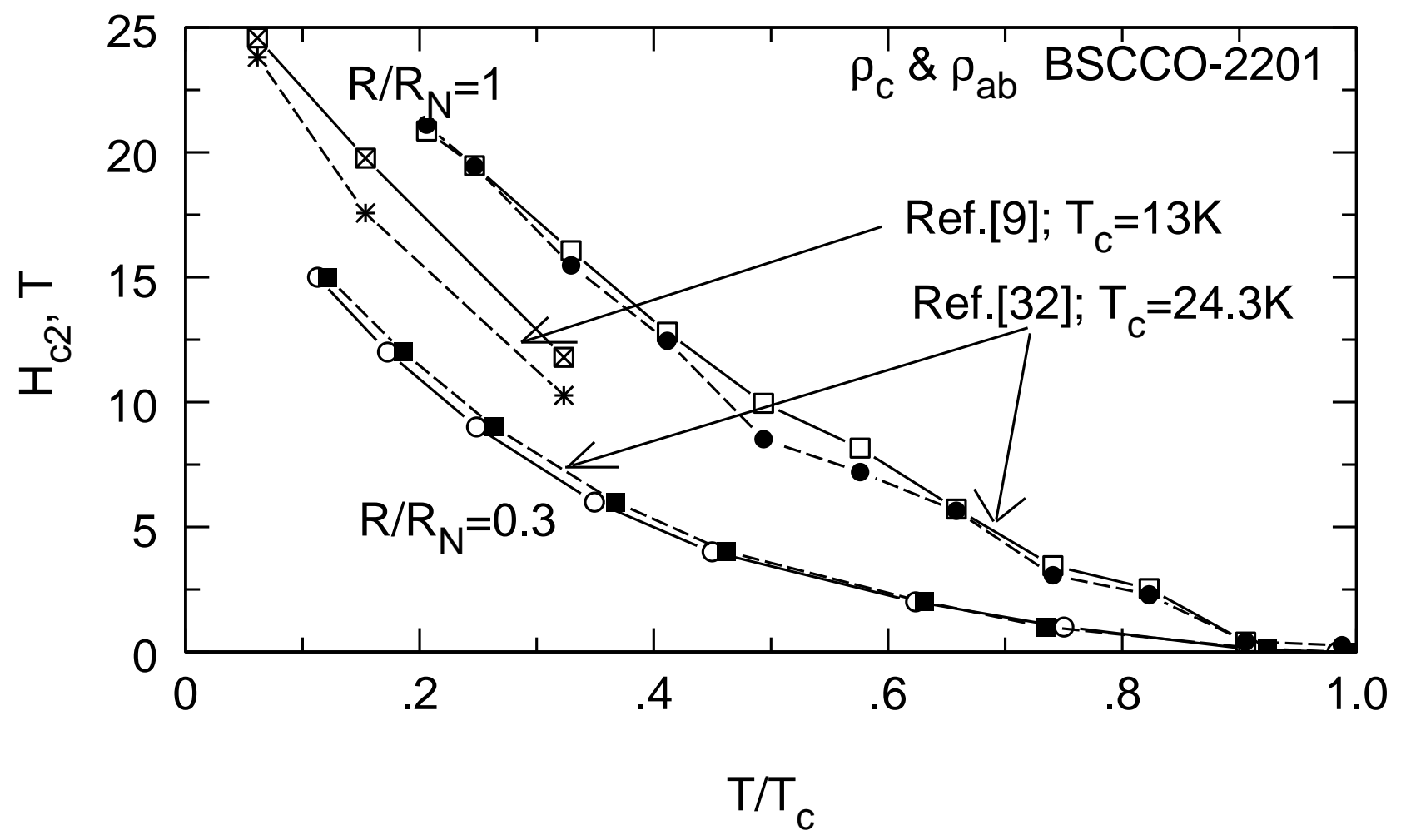

